\title{
Nonlinear effect of carrier drift on the performance of an n-type ZnO nanowire nanogenerator by coupling piezoelectric effect and semiconduction
}

\author{
Yuxing Liang ${ }^{1}$, Shuaiqi Fan ${ }^{1}$, Xuedong Chen ${ }^{2}$ and Yuantai Hu*1
}

\section{Full Research Paper}

\section{Address:}

${ }^{1}$ Department of Mechanics, Hubei Key Laboratory of Engineering Structural Analysis and Safety Assessment, Huazhong University of Science and Technology, Wuhan 430074, China and ${ }^{2}$ State Key Laboratory of Digital Manufacturing Equipment \& Technology, Huazhong University of Science and Technology, Wuhan 430074 , China

Email:

Yuantai Hu* - hudeng@263.net

${ }^{*}$ Corresponding author

\section{Keywords:}

carrier drift; crystallogrpahic c-axis; piezoelectric potential;

semiconductor; zinc oxide $(\mathrm{ZnO})$
Beilstein J. Nanotechnol. 2018, 9, 1917-1925. doi:10.3762/bjnano.9.183

Received: 13 January 2018

Accepted: 24 June 2018

Published: 04 July 2018

Associate Editor: N. Motta

(c) 2018 Liang et al.; licensee Beilstein-Institut. License and terms: see end of document.

\begin{abstract}
In piezoelectric semiconductors, electric fields drive carriers into motion/redistribution, and in turn the carrier motion/redistribution has an opposite effect on the electric field itself. Thus, carrier drift in a piezoelectric semiconducting structure is essentially nonlinear unless the induced fluctuation of carrier concentration is very small. In this paper, the nonlinear governing equation of carrier concentration was established by coupling both piezoelectric effect and semiconduction. A nonlinear carrier-drift effect on the performance of a $\mathrm{ZnO}$ nanogenerator was investigated in detail and it was elucidated that carrier motion/redistribution occurs in the $\mathrm{ZnO}$ nanowire (ZNW) cross section while there is no carrier motion in the axial direction. At the same time, we noted that the amplitude of boundary electric charge grows with increasing deformation, but the peaks of boundary electric charge do not appear at the cross-section endpoints. Thus, in order to effectively improve the performance of the ZNW nanogenerator, the effect of electrode configuration on the piezoelectric potential difference and output power was analyzed in detail. The electrode size for the optimal performance of a $\mathrm{ZnO}$ nanowire generator was proposed. This analysis that couples electromechanical fields and carrier concentration as a whole has some referential significance to piezotronics.
\end{abstract}

\section{Introduction}

An acoustic wave propagating in piezoelectric semiconductors usually stimulates electric fields that bring charge carriers into motion, and conversely, the carrier motion will produce an opposite effect on the electric fields and the acoustic wave itself [1-4]. This kind of interaction between an acoustic wave and carriers in piezoelectric semiconductors is called the acousto- 
electric effect, which is a special case of a more general phenomenon, called wave-particle drag [4,5]. Obviously, acoustoelectric coupling of piezoelectric semiconductors can be used to develop many new microelectronic devices with modern functions, for example piezoelectric field-effect transistors [6-11], piezoelectric charge-coupled devices [12-15], piezoelectric chemical sensors $[16,17]$, and nanogenerators made of vertically aligned $\mathrm{ZnO}$ nanowires [18-27]. The principle of nanogenerators is that the piezoelectric potential produced by the piezoelectric effect can produce a current in an external circuit when the ZNW is deformed. Specifically, a transversely applied force makes the nanowire bent when an atomic force microscopy tip scans over the top of the nanowire. The electromechanical coupling converts mechanical energy into electric energy [28,29]. A piezoelectric potential is built inside the nanowire with the stretched side being positively charged and the compressed side being negatively charged. At the same time, a Schottky barrier formed between the AFM tip and the nanowire and the piezoelectric potential will not disappear as long as the mechanical stress is maintained. This potential can be made use of to generate an electrical current [30].

Recently, Fan et al. studied the linear solutions of electromechanical quantities in a bent ZNW under the assumption of a small fluctuation of the carrier concentration [31]. Electric fields are proven to be independent of the axial position along the $c$-axis except near the end regions, and carrier motion/redistribution is proven to occur along the cross section. Because the semiconduction in ZNWs results in some electric leakage, a smaller initial carrier concentration is suggested to be more proper for energy-harvesting from a bent ZNW [31]. Because a small fluctuation of the carrier concentration implies a small deformation, a very low output of ZNW nanogenerators occurs under that situation. The force necessarily to obtain a good performance of the nanogenerator will certainly result in large variations of carrier concentration [30]. Thus, we abandon the assumption of small carrier-concentration fluctuations and establish the corresponding nonlinear governing equation of carrier concentration in this paper. The nonlinear accumulation of n-type carriers on one side of the ZNW cross section is shown in detail. Distribution characteristics of carrier concentration and electric potential in the cross section are discussed. Both the boundary electric charge and the boundary electric potential difference are calculated in depth. It is found that the amplitude of boundary electric charge always grows with increasing deformation, but the peaks of boundary electric charge do not appear directly at the cross-section endpoints. For harvesting a bent beam-like structure, the output electrodes are usually placed near the two cross section endpoints [30,32,33]. To improve the performance of a ZNW nanogenerator, the electrode configuration should be optimized with regard to piezo- electric potential difference and output power. We also carry out a detailed analysis on the effect of the electrode configuration.

\section{Nonlinear Governing Equation of Carrier Concentration for a Bent ZNW}

In a bent $\mathrm{ZnO}$ nanowire as shown in Figure 1, elastic fields can be solved in advance by using the irrotationality of static electric fields [31]. The two in-plane electric field components, $E_{1}$ and $E_{2}$, can be proven only to depend on $x_{1}$ and $x_{2}$, while the out-of-plane component $E_{3}$ is zero. The electric displacements, $\mathbf{D}=\left(D_{1}, D_{2}, D_{3}\right)^{\mathrm{T}}$, in the $\mathrm{ZNW}$ are:

$$
\begin{aligned}
& D_{1}=\alpha_{1} x_{1} x_{2}+\left(s_{44} e_{15}^{2}+\varepsilon_{11}\right) E_{1}, \\
& D_{2}=\alpha_{2} x_{1}^{2}-\alpha_{3}\left(a^{2}-x_{2}^{2}\right)+\left(s_{44} e_{15}^{2}+\varepsilon_{11}\right) E_{2}, \\
& D_{3}=-\alpha_{4} x_{2} x_{3},
\end{aligned}
$$

with

$$
\begin{aligned}
& \alpha_{1}=s_{44} e_{15}(P / 4 I)\left(1-2 s_{13} / s_{44}\right), \\
& \alpha_{2}=s_{44} e_{15}(P / 8 I)\left(1+6 s_{13} / s_{44}\right), \\
& \alpha_{3}=s_{44} e_{15}(P / 8 I)\left(3+2 s_{13} / s_{44}\right), \\
& \alpha_{4}=s_{33}(P / I)\left(e_{33}+2 e_{31} s_{13} / s_{33}\right),
\end{aligned}
$$

where $s_{K L}=\left(=c_{K L}^{-1}\right)$ are the compliance coefficients with $c_{K L}$ being the elastic constants; $\varepsilon_{i j}$ being the dielectric constants and $e_{i L}$ being the electromechanical coupling coefficients. $K$, $L=1,2, \ldots, 6 ; i, j=1,2,3 ; I=\int_{A} x_{2}^{2} \mathrm{~d} A$. The cross section of a bent $\mathrm{ZnO}$ nanowire is assumed to be circular in our analysis, which was also assumed by, for example, Gao and Wang [26,27], Henneghien et al. [34], and Maslov and co-workers [35]. In particular, Henneghien et al. have pointed out that circular and hexagonal nanowires exhibit the same behavior if the $\mathrm{ZnO}$ nanowire sections of each structure have the same area.

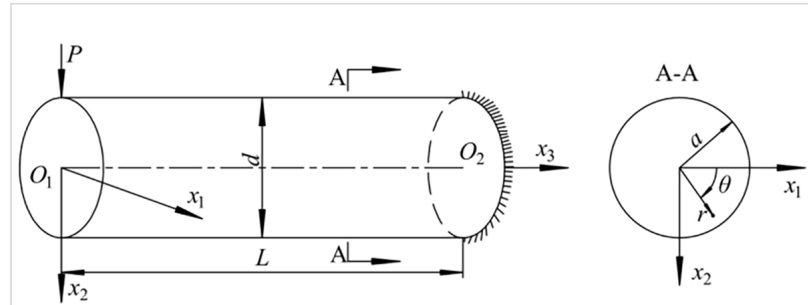

Figure 1: A circular ZNW cantilever exposed to a force $P$ at the free end. 
Linear solutions were obtained for small fluctuations of carrier concentration in a bent n-type $\mathrm{ZnO}$ nanowire under a force $P=0.7 \mathrm{nN}$ at the end of the nanowire [31]. However, small carrier concentration fluctuations, $\Delta n / n_{0} \ll 1$, where $\Delta n=n-n_{0}$, imply a very low output of the ZNW generator under consideration. Here, $n$ and $n_{0}$ stand for the actual and the initial carrier concentration, respectively. $P$ should be enlarged for obtaining a better performance of the nanogenerator. Electric fields appearing in piezoelectric semiconductors will move/ redistribute carriers and, in turn, the motion/redistribution of carriers will have an influence on the electric fields. This indicates that the first carrier drift term in the electric current expression, $J_{i}=q n \mu_{i j} E_{j}+q \kappa_{i j} n_{, j}$, is essentially nonlinear. Since a ZNW with the crystallographic $c$-axis along the $x_{3}$-direction is transversely isotropic, the electron mobility $\mu_{i j}$ and the diffusion coefficients $\kappa_{i j}$ can be written as

$$
\begin{aligned}
& \left(\mu_{i j}\right)=\operatorname{diag}\left(\mu_{11}, \mu_{11}, \mu_{33}\right), \\
& \left(\kappa_{i j}\right)=\operatorname{diag}\left(\kappa_{11}, \kappa_{11}, \kappa_{33}\right) .
\end{aligned}
$$

The two in-plane components of the electric field can thus be obtained from $J_{i}=q n \mu_{i j} E_{j}+q \kappa_{i j} n_{, j}=0$, the null-current condition, as follows with $\zeta=\kappa_{11} / \mu_{11}$ :

$$
\begin{aligned}
& E_{1}=-\zeta \frac{1}{n} \frac{\partial n}{\partial x_{1}} \\
& E_{2}=-\zeta \frac{1}{n} \frac{\partial n}{\partial x_{2}}
\end{aligned}
$$

Substituting Equation 4 into Equation 1 and then into the Gauss law, $\nabla \cdot \mathrm{D}=-q \Delta n$, yields the nonlinear governing equation of carrier concentration $\bar{n}=n / n_{0}$ as

$$
\begin{aligned}
m_{2} \bar{x}_{2}= & \frac{1}{\bar{n}}\left(\frac{\partial^{2} \bar{n}}{\partial \bar{x}_{1}^{2}}+\frac{\partial^{2} \bar{n}}{\partial \bar{x}_{2}^{2}}\right) \\
& -\frac{1}{\bar{n}^{2}}\left[\left(\frac{\partial \bar{n}}{\partial \bar{x}_{1}}\right)^{2}+\left(\frac{\partial \bar{n}}{\partial \bar{x}_{2}}\right)^{2}\right] \\
& +m_{1} n_{0}(\bar{n}-1)
\end{aligned}
$$

where $\left(\bar{x}_{1}, \bar{x}_{2}\right)=\left(x_{1} / a, x_{2} / a\right)$ and

$$
\begin{aligned}
& m_{1}=q / k_{1}, k_{1}=-\zeta\left(s_{44} e_{15}^{2}+\varepsilon_{11}\right) \\
& m_{2}=-\left(P / I k_{1}\right)\left(e_{15} s_{44}-2 e_{31} s_{13}-e_{33} s_{33}\right) .
\end{aligned}
$$

In the vacuum outside the $\mathrm{ZNW}$ cross-section, the electric potential $\phi^{-}$should satisfy

$$
\nabla^{2} \phi^{-}=0
$$

The point of zero electric potential is set at infinity, i.e., $\left.\phi^{-}\right|_{\infty}=0$. The continuous conditions of normal electric displacement $D_{r}^{ \pm}$and electric potential $\phi^{ \pm}$at the boundary $\Omega$, $r=a$, of the $\mathrm{ZNW}$ cross section require

$D_{r}^{+}=\left(D_{1}, D_{2}, D_{3}\right) \cdot(\cos \theta, \sin \theta, 0)^{\mathrm{T}}=-\varepsilon_{0} \phi_{, r}^{-}, \phi^{+}=\phi^{-},(8)$

where $\phi^{+}$refers to the electric potential within the ZNW cross section, and $r$ and $\theta$ stand for the radial and the tangential coordinate, respectively, as shown in Figure 1. $\varepsilon_{0}$ is the dielectric coefficient of the vacuum.

\section{Results and Discussion}

We calculate carrier concentration fluctuation, piezoelectric potential, electric fields, boundary charges by using the finite element method for $P$ from $0.7 \mathrm{nN}$ to $80 \mathrm{nN}$, where the $\mathrm{ZnO}$ nanowire has a diameter $d=50 \mathrm{~nm}$ and its $c$-axis is oriented along the $x_{3}$-direction. The material constants are given below in Equation 9 [36-41] with $\varepsilon_{0}=8.8542 \cdot 10^{-12} \mathrm{~F} / \mathrm{m}$.

For a bent n-type ZNW in the linear regime, a positive piezoelectric potential appears at the stretched side and negative piezoelectric potential appears at the compressed side [26-31], i.e., the electric potential is high at $\bar{x}_{2}<0$ and low at $\bar{x}_{2}>0$. When the end force $P$ increases, n-type carriers will be driven to drift upwards with an accumulation at the stretched side, $\Delta n / n_{0}$, becoming so large that the linear balance regime collapses. Non-uniformity in carrier concentration accompanied by drift will bring about also diffusion. These two effects of drift and diffusion will achieve a new equilibrium at every step of

$$
\begin{aligned}
& \left(c_{11}, c_{12}, c_{13}, c_{33}, c_{44}, c_{55}\right)=(207,117.7,106.1,209.5,44.8,44.6) \mathrm{GPa}, \\
& \left(e_{31}, e_{33}, e_{15}\right)=(-0.51,1.22,-0.45) \mathrm{C} / \mathrm{m}^{2},\left(\varepsilon_{11}, \varepsilon_{33}\right)=(7.77,8.91) \varepsilon_{0} \\
& \kappa_{11}=5.2 \cdot 10^{-4} \mathrm{~m}^{2} / \mathrm{s}, \mu_{11}=0.02 \mathrm{~m}^{2} / \mathrm{Vs} .
\end{aligned}
$$


increase in $P$. A higher accumulation of carriers in $\bar{x}_{2}<0$ results in a stronger nonlinearity. Figure $2 \mathrm{a}$ shows the nonlinearity manifesting itself from carrier drift with $n_{0}=1.0 \cdot 10^{23} \mathrm{~m}^{-3}$ for $P=0.7,1.5,3.0,5.0$ and $10.0 \mathrm{nN} . P=0.7 \mathrm{nN}$ corresponds to the linear case of which the largest variance of carrier concentration, $\left|\partial \bar{n} / \partial \bar{x}_{2}\right|$, appears along the neutral axis [31]. There are two factors to affect $\left|\partial \bar{n} / \partial \bar{x}_{2}\right|$ : mechanical shear deformations and accumulation of electric carriers. In general, shear deformations result in in-plane electric fields, which lead to carrier drift, while carrier accumulation results in diffusion. Because $\Delta n / n_{0}$ is very small in the whole ZNW cross section in the linear regime, the largest variance rate of carrier concentration appears along the neutral axis because of the strongest shear deformation there. With increasing end force, the carrier accumulation increases such that there is a stronger nonlinear drift effect on $\partial \bar{n} / \partial \bar{x}_{2}=-\bar{n} E_{2} / \zeta$. The carrier concentration gradually grows in the upper portion and reduces in the lower portion, which yields $\bar{n}>1.0$ for $\bar{x}_{2}<0$ and $\bar{n}<1.0$ for $\bar{x}_{2}>0$. Hence, at larger $P,\left|\partial \bar{n} / \partial \bar{x}_{2}\right|$ becomes larger for $\bar{x}_{2}<0$ and smaller for $\bar{x}_{2}>0$ and the position of the largest variance $\left|\partial \bar{n} / \partial \bar{x}_{2}\right|$ gradually shifts upwards, as shown in Figure 2a. When $P$ becomes very large (Figure $2 \mathrm{~b}, P=10,30,50,60,70$ and $80 \mathrm{nN}$ for $n_{0}=1.0 \cdot 10^{23} \mathrm{~m}^{-3}$ ), the position of the largest variance shifts upwards even more, implying that most of the carriers are accumulated in the upper cross section.

Figure 3 shows the carrier distribution in the ZNW cross section for $P=50,60,70$ and $80 \mathrm{nN}$. It is easy to find numerically that $\int_{A}\left(\Delta n / n_{0}\right) \mathrm{d} A \neq 0$. Given the charge-balance condition this results needs to be explained. It is very obvious that there is no drift and diffusion of carriers along the axial $x_{3}$-direction due to $E_{3}=0$. Carrier drift and diffusion only occur along the cross section. In thermal equilibrium, the Fermi energy level $\Sigma_{\mathrm{f}}$ must be consistent in the cross section with the carrier concentration satisfying

$$
n\left(x_{1}, x_{2}\right)=n_{i} \exp \left[\left(\sum_{\mathrm{f}}-\sum_{i}\left(x_{1}, x_{2}\right)\right) / k_{\mathrm{B}} T\right]
$$

where $n_{i}$ is the intrinsic carrier concentration of $\mathrm{ZnO} ; \Sigma_{i}\left(x_{1}, x_{2}\right)$ stands for the intrinsic energy level that can be affected by the electric potential field; $k_{\mathrm{B}}$ is the Boltzmann constant and $T$ is the temperature (300 $\mathrm{K}$ in our analysis). $n_{0}=n_{i} \cdot \exp \left[\left(\Sigma_{\mathrm{f}}-\Sigma_{i}(0)\right) / k_{\mathrm{B}} T\right], \Sigma_{i}(0)$ is the initial intrinsic energy level, which is constant in the whole cross section under $P=0$. When the ZNW is bent by a nonzero end force $P, \Sigma_{i}\left(x_{1}, x_{2}\right)$ becomes alterable in the cross section in terms of the electric potential field, which turns Equation 10 into

$$
\begin{aligned}
n\left(x_{1}, x_{2}\right) & =n_{i} \exp \left\{\left[\sum_{\mathrm{f}}-\left(\sum_{i}(0)-q \phi\right)\right] / k_{\mathrm{B}} T\right\} \\
& =n_{0} \exp \left[q \phi\left(x_{1}, x_{2}\right) / k_{\mathrm{B}} T\right] .
\end{aligned}
$$

Equation 11 indicates an exponential relationship between carrier concentration and electric potential in the cross section. Figure 4 shows the distribution of the electric potential in the ZNW cross section for $P=50,60,70$ and $80 \mathrm{nN}$, which can also be obtained directly from Figure 3 by using Equation 11. In the electric potential field, $\phi\left(x_{1}, x_{2}\right)$, all n-type carriers in the cross section obtain additional electric potential energy, $q \phi\left(x_{1}, x_{2}\right)$. This potential energy increases/reduces the energy of electrons there, and thus, increases/decreases the number of the n-type carriers according to Equation 11, i.e., it is the appearance of additional electric potential energy induced by the electric potential field that results in $\int_{A}\left(\Delta n / n_{0}\right) \mathrm{d} A \neq 0$.

It follows from Figure 4 that there is a strong electric potential gradient along the $x_{2}$-direction, and the maximal electric potential difference is between the two endpoints of the $x_{2}$-axis. Furthermore, the maximal positive potential amplitude is much

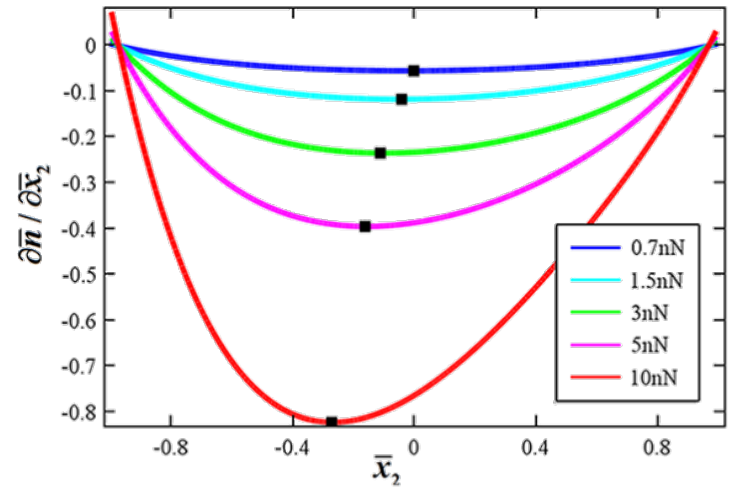

(a)

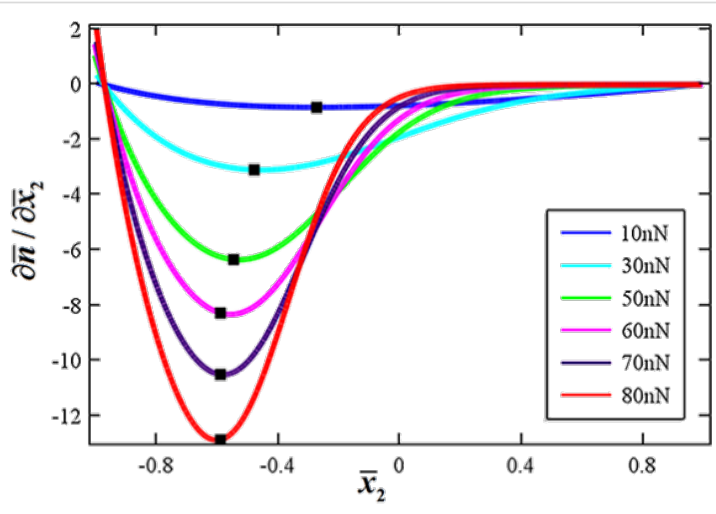

(b)

Figure 2: Nonlinearity as a result of carrier drift for $n_{0}=1.0 \cdot 10^{23} \mathrm{~m}^{-3}$ as a function of the end force $P$. a) $P=0.7,1.5,3.0,5.0$ and $10.0 \mathrm{nN}$; b) $P=10$, $30,50,60,70$ and $80 \mathrm{nN}$. 

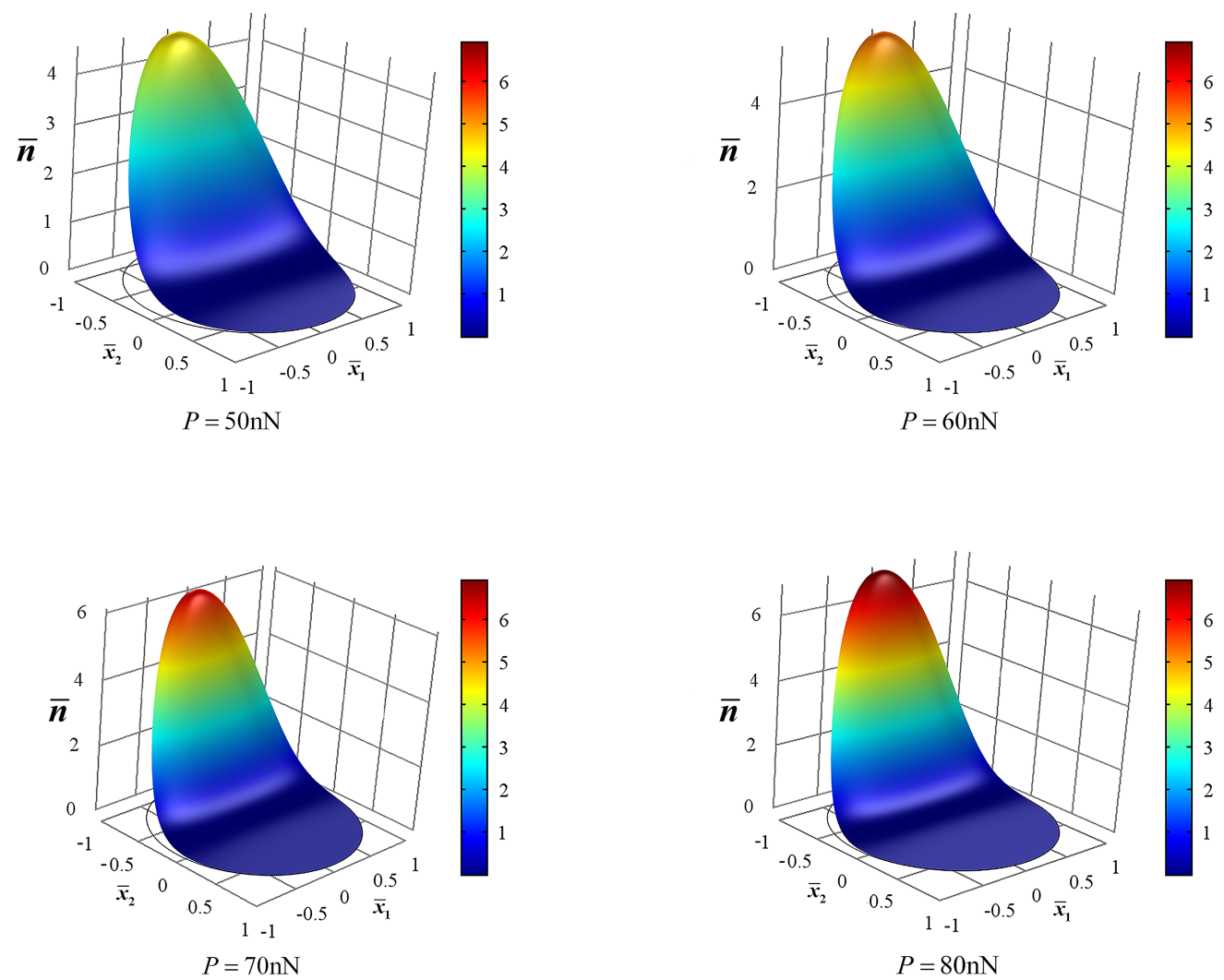

Figure 3: Carrier distribution in the ZNW cross section for $P=50,60,70$ and $80 \mathrm{nN}$.
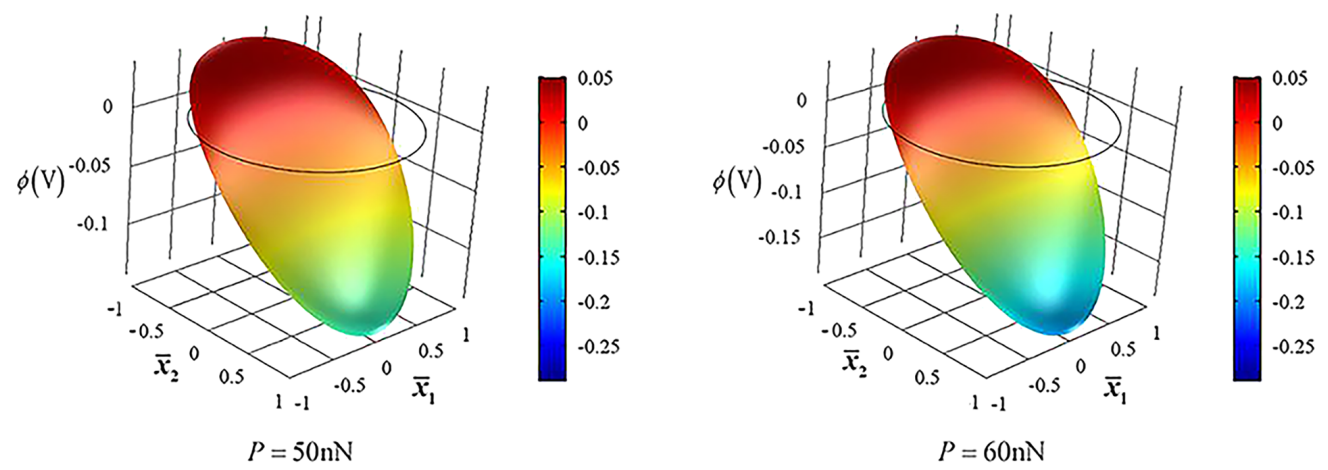

$P=60 \mathrm{nN}$
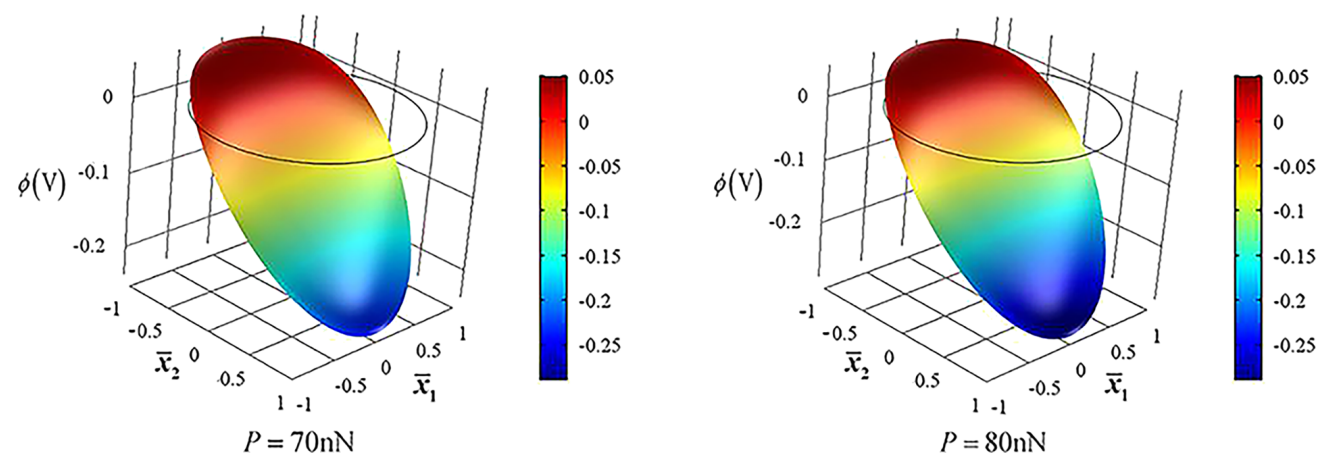

Figure 4: Distribution of electric potential in the ZNW cross section for $P=50,60,70$ and $80 \mathrm{nN}$. 
lower than the maximal negative potential amplitude. In a bent piezoelectric semiconducting beam, two shear deformations produce two in-plane electric field components, $E_{1}$ and $E_{2} . E_{1}$ is induced by the shear strain $S_{5}$ in the $x_{1}-x_{3}$ plane and $E_{2}$ is induced by the shear strain $S_{4}$ in the $x_{2}-x_{3}$ plane. When $P$ acts along the $x_{2}$-direction, $\sigma_{5}\left(\tau_{13}\right)$ is very small, and so is $S_{5}$. Thus, both $\sigma_{5}$ and $S_{5}$ produce negligible influence on electric field, carrier concentration and electric potential. $S_{4}$ is the primary deformation component to induce the electric field $E_{2}$ and carrier redistribution. We show in Figure 5 the effect of $n_{0}$ on $E_{2}$ in order to understand why the maximal positive potential amplitude is much less than the maximal negative potential amplitude [27]. For comparison, we have also included the electric field $E_{2}$ induced only by the piezoelectric effect of a $\mathrm{ZNW}$ without taking into account semiconduction (marked as "piezo" in the following figures). Independently of the deformation, in a piezoelectric insulator ZNW (without semiconduction) its Fermi energy level is always at the center of the forbidden band. Hence, the electric field $E_{2}$ induced only by the piezoelectric characteristics is symmetrical with regard to the neutral axis, as shown in Figure 5. After n-type doping, the Fermi level moves upwards from the center of the forbidden band. The deformation-induced electric field induces motion/redistribution of carriers: the positive electric field $E_{2}$ drives the carriers upwards, i.e., the bottom portion, $\bar{x}_{2}>0$, loses carriers and the upper portion, $\bar{x}_{2}<0$, accumulates carriers. This motion/redistribution of carriers will in turn decrease the positive electric field $E_{2}$. Moreover, $E_{2}$ is reduced more at $\bar{x}_{2}<0$ than at $\bar{x}_{2}>0$. It can be found from Figure 3 that $\bar{n}-1=0$ occurs at $\bar{x}_{2}{ }^{*} \approx$ $-0.236,-0.273,-0.309$ and -0.338 for $P=50,60,70$ and $80 \mathrm{nN}$, respectively, with $n_{0}=1.0 \cdot 10^{23} \mathrm{~m}^{-3}$. It should be noted that at $\bar{x}_{2}{ }^{*}, \phi=0$ and the intrinsic energy level is $\Sigma_{i}(0)$. Because $E_{2}=-\partial \phi / \partial x_{2}$ is reduced more in the region $-1 \leq \bar{x}_{2} \leq \bar{x}_{2}{ }^{*}$ than in $\bar{x}_{2}{ }^{*} \leq \bar{x}_{2} \leq 1$, as shown in Figure 5, the amplitude of the positive electric potential at $\bar{x}_{2}=-1$ becomes much smaller than the amplitude of the negative electric potential at $\bar{x}_{2}=1$. For example, for $P=80 \mathrm{nN}$, the maximal positive electric potential at $\bar{x}_{2}=-1$ is about $0.05 \mathrm{~V}$ and the maximal negative potential at $\bar{x}_{2}=1$ is about $0.3 \mathrm{~V}$, which is in agreement with the experimental results described on page 36 of [30].

We now introduce $\eta=n_{0} / n^{\prime}$ with $n^{\prime}=1 \cdot 10^{20} \mathrm{~m}^{-3}$. Figure 6 shows effect of the initial carrier concentration $n_{0}$ on the output voltage $V_{\text {out }}=\left.\phi\right|_{x_{2}=-1}-\left.\phi\right|_{x_{2}=1}$ between the two endpoints of the $x_{2}$-axis of a bent $\mathrm{ZNW}$ cross section for $P=50,60,70$ and $80 \mathrm{nN}$, respectively. We have included in Figure 6 four horizontal lines for comparison, corresponding to the four output voltages calculated only from the pure piezoelectric effect without taking semiconduction into account. For a low initial carrier concentration, for example $\eta<0.5$, the Fermi energy level is a little bit above the center of the forbidden band. Thus, the output voltage is very similar to that of the purely piezoelectric effect. For higher initial carrier concentrations, e.g., $\eta>4.5$, the Fermi energy level is far higher than the center of the forbidden
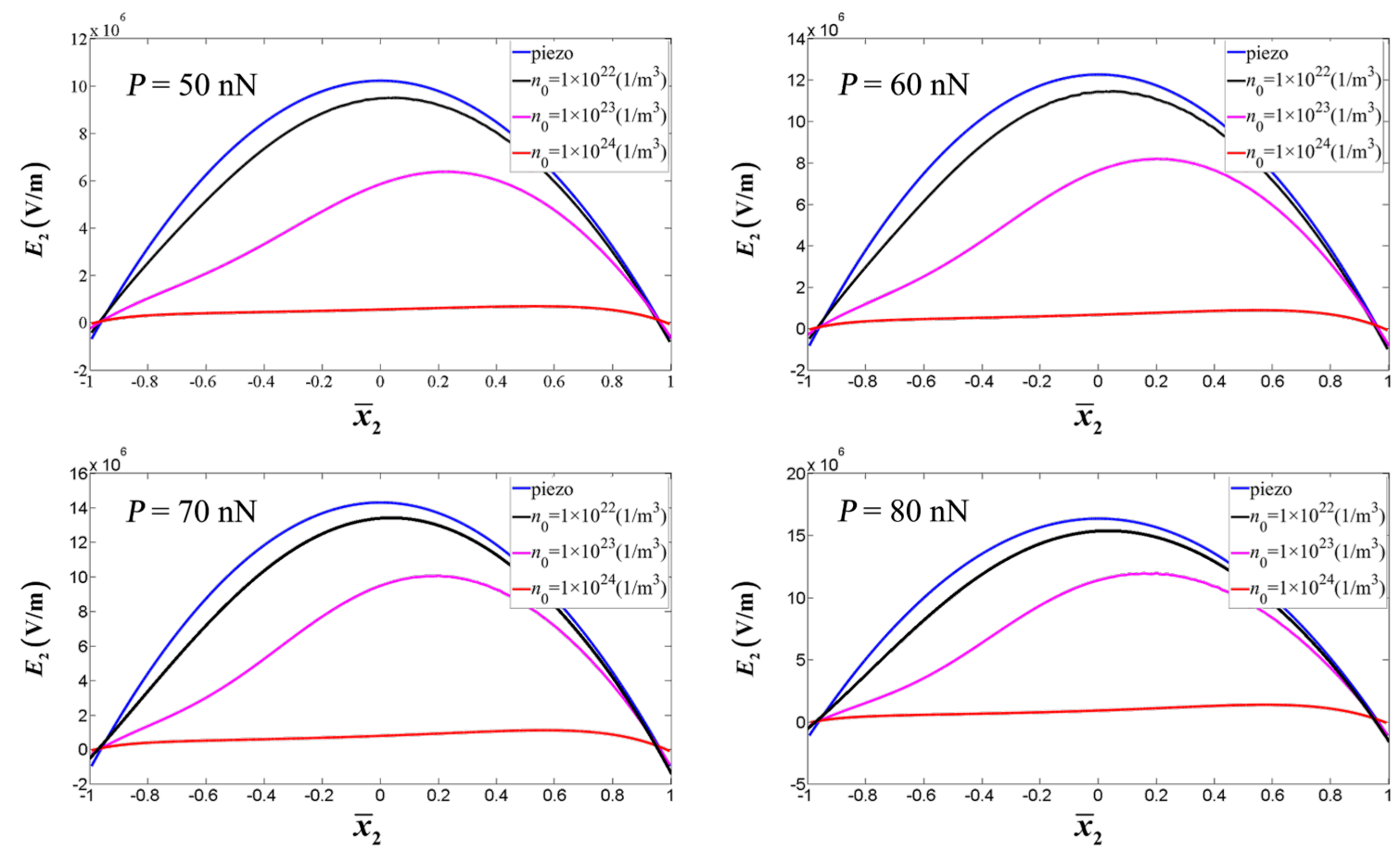

Figure 5: Effect of initial carrier concentration $n_{0}$ on the electric field $E_{2}$ along the $x_{2}$-axis. 


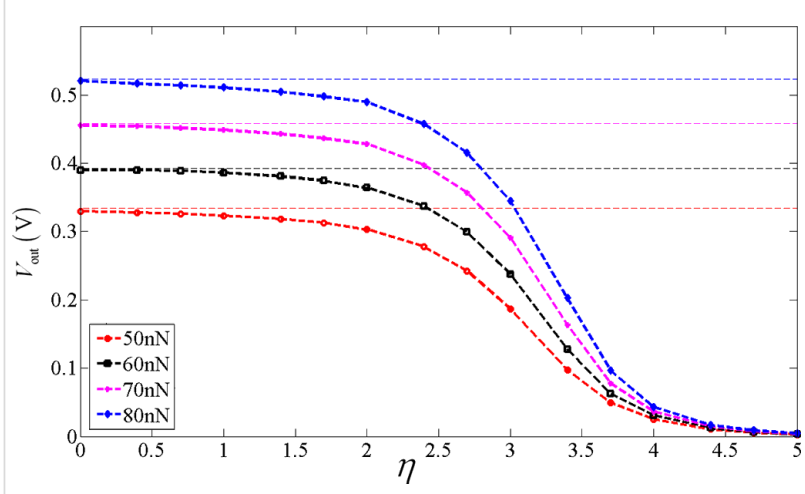

Figure 6: Output voltage $V_{\text {out }}$ between the two endpoints of the cross section of a bent ZNW as a function of the initial carrier concentration.

band. Hence, the output voltage becomes very low. This phenomenon indicates again that a smaller initial carrier concentration is more proper for energy harvesting with a bent $\mathrm{ZNW}$ [31].

Figure $7 \mathrm{a}$ shows the distributions of the boundary electric potential for different end forces with $n_{0}=1 \cdot 10^{23} \mathrm{~m}^{-3}$. The boundary electric potential reaches the minimum and the maximum at $\theta=\pi / 2$ and $\theta=3 \pi / 2$, respectively, which indicates that the maximal electric potential difference $V_{\text {out }}$ occurs between the endpoints at top and bottom. Furthermore, $V_{\text {out }}$ increases with increasing $P$. Figure $7 \mathrm{~b}$ shows the boundary electric displacement at $\Omega$ as a function of the end force. We note that with an increase in end force $P$, the maximal boundary electric displacement does no longer appear at the upper endpoint of the $x_{2}$-axis. Instead, there are two peaks in the region $\pi<\theta<2 \pi$ with a certain angular deviation from the endpoint. The appearance of this phenomenon is due to the excessive nonlinear accumulation of carriers in the upper part of the cross section. The boundary charge is quite large between these two peaks and should be collected. Thus, the design of an effective electrode configuration becomes a significant issue in order to improve the output of a $\mathrm{ZnO}$ nanogenerator.

Consider the electrode configuration in the inset of Figure 8. We repeat the numerical calculation by dividing the boundary into two parts: one is the continuous boundary (outside the electrodes) and the other is constant electric potential boundary (inside the electrodes). In this situation, the electric charge $Q_{e}$ at the electrode can be obtained $[42,43]$ through the integral of the boundary electric displacement $D_{r}$ over the distributed surface $3 \pi / 2-\gamma<\theta<3 \pi / 2+\gamma$,

$$
Q_{e}=\int_{-\gamma}^{\gamma} D_{r}(a, 3 \pi / 2+v) a \mathrm{~d} v
$$

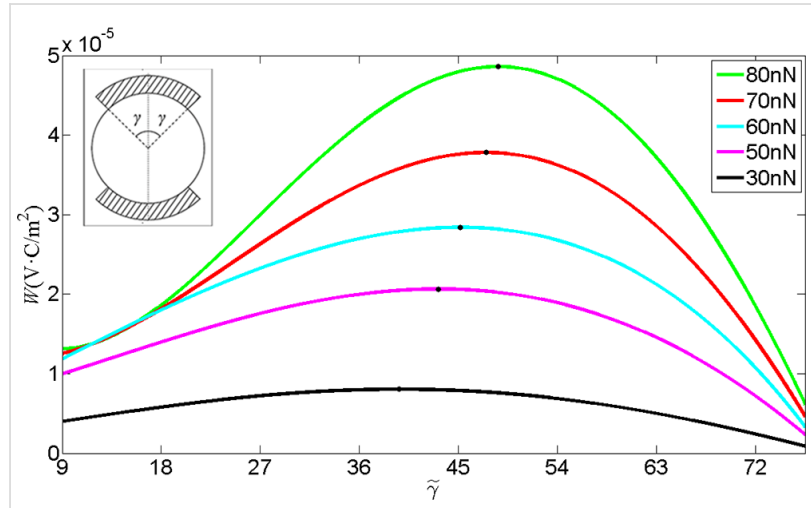

Figure 8: $W$ as a function of the electrode configuration for different end forces.

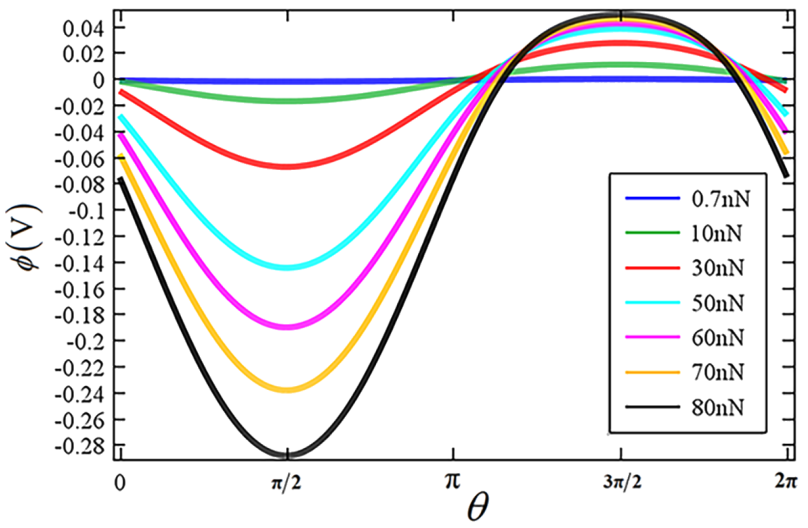

(a)

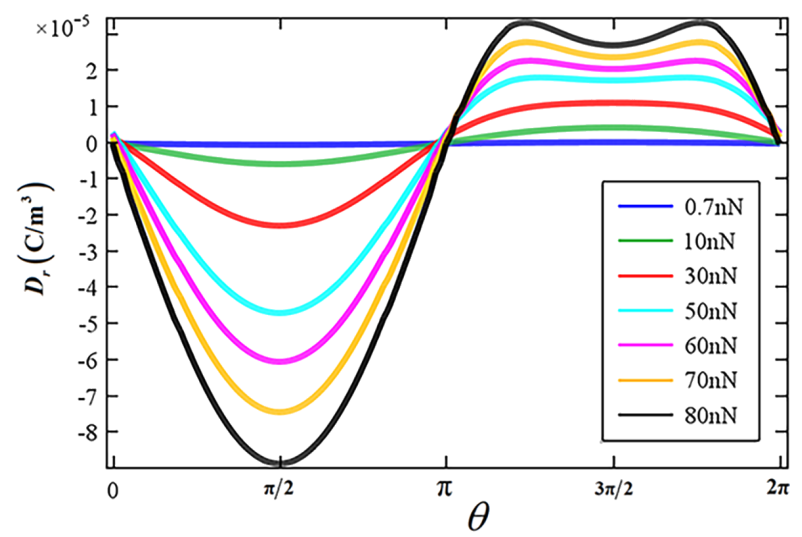

(b)

Figure 7: (a) Boundary electric potential $\phi$ and (b) boundary electric displacement $D_{r}$ at $\Omega$ for different end forces $P=0.7,10,30,50,60,70$ and $80 \mathrm{nN}$. 
The current flowing through this electrode is $I_{e}=-\dot{Q}_{e}$, which indicates that the output current $I_{e}$ for a harmonic vibration is directly proportional to the amplitude of the boundary electric charge $Q_{e}$. Thus, for convenience we define the quantity $W$,

$$
W=\int_{-\gamma}^{\gamma} D_{r}(a, 3 \pi / 2+v) a \mathrm{~d} v \cdot V_{\text {out }}
$$

which is directly proportional to the output power of the ZNW generator. $V_{\text {out }}=\phi^{+}\left(a, \theta_{0}\right)-\phi^{+}\left(a, 2 \pi-\theta_{0}\right)$ stands for the electric potential difference between the two electrodes and $\theta_{0}=\gamma+3 \pi / 2$. Figure 8 shows $W$ as a function of $\tilde{\gamma}$, with $\tilde{\gamma}=(180 / \pi) \gamma$. With $P$ changing from 30 to $80 \mathrm{nN}$, the peak point of $W$ moves from $\theta_{0}=1.72 \pi\left(\tilde{\gamma} \approx 40^{\circ}\right)$ to $\theta_{0}=1.77 \pi$ $\left(\tilde{\gamma} \approx 49^{\circ}\right)$. Obviously, a flare angle of $2 \tilde{\gamma} \approx 98^{\circ}$ of the two electrodes is suitable for all loadings below $80 \mathrm{nN}$. This electrode configuration provides an optimal output of the nanogenerator.

In addition, we show the effect of the initial carrier concentration on the optimal electrode configuration in Figure 9 for $n_{0}=1 \cdot 10^{22}, 1 \cdot 10^{23}$ and $1 \cdot 10^{24} \mathrm{~m}^{-3}$. Obviously, a smaller initial carrier concentration is corresponding to a higher energy harvest. It is readily understood that semiconduction results in some electric leakage, and thus reduces the output power. For different initial carrier concentrations, electrode configurations with flare angles of $2 \tilde{\gamma} \approx 88^{\circ}, 98^{\circ}$ and $88^{\circ}$ are suitable for a ZNW generator with $n_{0}=1 \cdot 10^{22}, 1 \cdot 10^{23}$ and $1 \cdot 10^{24} \mathrm{~m}^{-3}$, respectively.

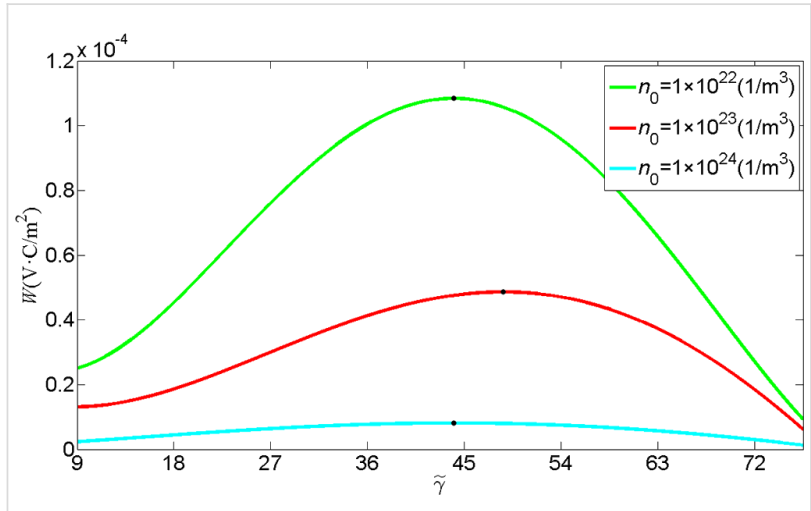

Figure 9: $W$ as a function of the electrode configuration for different initial carrier concentrations.

\section{Conclusion}

Nonlinear solutions for carrier concentration, electric field and electric potential in a bent ZNW are obtained without the assumption of small fluctuations of carrier concentration. As the bending deformation increases, carriers are gradually accumulated in the stretched portion of the ZNW, and the boundary charge is greatly increased. It is found that the electrode configuration will have a large influence on the output performance of a bent ZNW generator. Thus it is of significance to design an electrode configuration for an optimal output. Both the analysis technique and the obtained results are useful in the design of piezotronics and piezo-phototropic devices and the corresponding applications.

\section{Acknowledgements}

This work was supported by the National Natural Science Foundation of China (Grant Nos. 11672113 and 51435006) and the Key Laboratory Project of Hubei Province (No. 2016CFA073). Many thanks to Professor Yunbo Wang at School of Optical and Electronic Information of Huazhong University of Science and Technology for the helpful discussions and suggestions.

\section{ORCID ${ }^{\circledR}$ iDs}

Yuxing Liang - https://orcid.org/0000-0003-4120-2277

\section{References}

1. Hutson, A. R.; White, D. L. J. Appl. Phys. 1962, 33, 40-47. doi:10.1063/1.1728525

2. White, D. L. J. Appl. Phys. 1962, 33, 2547-2554. doi:10.1063/1.1729015

3. Collins, J. H.; Lakin, K. M.; Quate, C. F.; Shaw, H. J. Appl. Phys. Lett. 1968, 13, 314-316. doi:10.1063/1.1652628

4. Yang, J. S.; Zhou, H. G. Int. J. Solids Struct. 2005, 42, 3171-3183. doi:10.1016/j.ijsolstr.2004.10.011

5. Weinreich, G.; Sanders, T. M., Jr.; White, H. G. Phys. Rev. 1959, 114 , 33-44. doi:10.1103/PhysRev.114.33

6. Zhang, Y.; Liu, Y.; Wang, Z. L. Adv. Mater. 2011, 23, 3004-3013. doi:10.1002/adma.201100906

7. Dahiya, R. S.; Metta, G.; Valle, M.; Adami, A.; Lorenzelli, L. Appl. Phys. Lett. 2009, 95, 034105. doi:10.1063/1.3184579

8. Wu, Y.-R.; Singh, J. Appl. Phys. Lett. 2004, 85, 1223-1225. doi:10.1063/1.1784039

9. Wu, W.; Pan, C.; Zhang, Y.; Wen, X.; Wang, Z. L. Nano Today 2013, 8, 619-642. doi:10.1016/j.nantod.2013.11.002

10. Wang, Z. L. Mater. Sci. Eng., R 2009, 64, 33-71. doi:10.1016/j.mser.2009.02.001

11. Mante, P.-A.; Huang, Y.-R.; Yang, S.-C.; Liu, T.-M.; Maznev, A. A.; Sheu, J.-K.; Sun, C.-K. Ultrasonics 2015, 56, 52-65. doi:10.1016/j.ultras.2014.09.020

12. Willatzen, M.; Christensen, J. Phys. Rev. B 2014, 89, 041201. doi:10.1103/PhysRevB.89.041201

13. Gokhale, V. J.; Rais-Zadeh, M. Sci. Rep. 2014, 4, 5617. doi:10.1038/srep05617

14. Trotta, R.; Wildmann, J. S.; Zallo, E.; Schmidt, O. G.; Rastelli, A. Nano Lett. 2014, 14, 3439-3444. doi:10.1021/nl500968k

15. Yakovenko, V. M. Physica B 2012, 407, 1969-1972. doi:10.1016/j.physb.2012.01.076

16. Fan, L.; Chen, Z.; Zhang, S.-y.; Zhang, H. J. Appl. Phys. 2014, 115, 064506. doi:10.1063/1.4865172

17. Shim, Y.-S.; Zhang, L.; Kim, D. H.; Kim, Y. H.; Choi, Y. R.; Nahm, S. H.; Kang, C.-Y.; Lee, W.; Jang, H. W. Sens. Actuators, B 2014, 198, 294-301. doi:10.1016/j.snb.2014.03.073 
18. Qin, Y.; Wang, X.; Wang, Z. L. Nature 2008, 451, 809-813. doi:10.1038/nature06601

19. Yang, R. S.; Qin, Y.; Dai, L.; Wang, Z. L. Nat. Nanotechnol. 2009, 4, 34-39. doi:10.1038/nnano.2008.314

20. Xu, S.; Qin, Y.; Xu, C.; Wei, Y.; Yang, R.; Wang, Z. L. Nat. Nanotechnol. 2010, 5, 366-373. doi:10.1038/nnano.2010.46

21. Zhu, G.; Yang, R.; Wang, S.; Wang, Z. L. Nano Lett. 2010, 10 3151-3155. doi:10.1021/nl101973h

22. Wang, Z. L. Adv. Funct. Mater. 2008, 18, 3553-3567. doi:10.1002/adfm.200800541

23. Wang, Z. L.; Yang, R.; Zhou, J.; Qin, Y.; Xu, C.; Hu, Y.; Xu, S. Mater. Sci. Eng., R 2010, 70, 320-329. doi:10.1016/j.mser.2010.06.015

24. Wang, Z. L. Nanogenerators for Self-powered Devices and Systems, Georgia Institute of Technology: Atlanta, GA, U.S.A., 2011. https://smartech.gatech.edu/handle/1853/39262

25. Wang, Z. L. Physics (China) 2006, 35, 897-903

26. Gao, Y.; Wang, Z. L. Nano Lett. 2007, 7, 2499-2505. doi:10.1021/nl071310j

27. Gao, Y.; Wang, Z. L. Nano Lett. 2009, 9, 1103-1110. doi:10.1021/nl803547f

28. Wang, Z. L.; Song, J. Science 2006, 312, 242-246. doi:10.1126/science.1124005

29. Sun, C.; Shi, J.; Wang, X. J. Appl. Phys. 2010, 108, 034309. doi:10.1063/1.3462468

30. Wang, Z. L. Piezotronics and Piezo-Phototronics; Springer: Berlin, Germany, 2012. doi:10.1007/978-3-642-34237-0

31. Fan, S.; Liang, Y.; Xie, J.; Hu, Y. Nano Energy 2017, 40, 82-87. doi:10.1016/j.nanoen.2017.07.049

32. Xie, J.; Yang, J.; Hu, H.; Hu, Y.; Chen, X. J. Intell. Mater. Syst. Struct. 2012, 23, 135-139. doi:10.1177/1045389X11431744

33. Wang, H.-r.; Xie, J.-m.; Xie, X.; Hu, Y.-t.; Wang, J. Appl. Math. Mech. (Engl. Ed.) 2014, 35, 229-236. doi:10.1007/s10483-014-1786-6

34. Henneghien, A.-L.; Gayral, B.; Désières, Y.; Gérard, J.-M. J. Opt. Soc. Am. B 2009, 26, 2396-2403. doi:10.1364/JOSAB.26.002396

35. Maslov, A. V.; Bakunov, M. I.; Ning, C. Z. J. Appl. Phys. 2006, 99 , 024314. doi:10.1063/1.2164538

36. Yang, J. An Introduction to the Theory of Piezoelectricity; Springer: New York, NY, U.S.A., 2005. doi:10.1007/b101799

37. Kobiakov, I. B. Solid State Commun. 1980, 35, 305-310. doi:10.1016/0038-1098(80)90502-5

38. Carlotti, G.; Socino, G.; Petri, A.; Verona, E. Appl. Phys. Lett. 1987, 51, 1889-1891. doi:10.1063/1.98502

39. Zhang, C.; Wang, X.; Chen, W.; Yang, J. Smart Mater. Struct. 2017, 26, 025030. doi:10.1088/1361-665X/aa542e

40. Hu, Y.; Zeng, Y.; Yang, J. Int. J. Solids Struct. 2007, 44, 3928-3938. doi:10.1016/j.jjsolstr.2006.10.033

41. Luo, Y.; Cheng, R.; Zhang, C.; Chen, W.; Yang, J. Acta Mech. Solida Sin. 2018, 31, 127-140. doi:10.1007/s10338-018-0010-1

42. Yang, J. S.; Fang, H. Y. IEEE Trans. Ultrason., Ferroelectr., Freq. Control 2002, 49, 798-804. doi:10.1109/TUFFC.2002.1009338

43. Yang, J. Acta Mech. Solida Sin. 2017, 30, 21-26. doi:10.1016/j.camss.2016.05.001

\section{License and Terms}

This is an Open Access article under the terms of the Creative Commons Attribution License (http://creativecommons.org/licenses/by/4.0), which permits unrestricted use, distribution, and reproduction in any medium, provided the original work is properly cited.

The license is subject to the Beilstein Journal of Nanotechnology terms and conditions: (https://www.beilstein-journals.org/bjnano)

The definitive version of this article is the electronic one which can be found at:

doi:10.3762/bjnano.9.183 